\title{
Differential expression profiles of the Hedgehog signaling pathway between microsatellite-stable and microsatellite-unstable colorectal cancers
}

\author{
XUGUANG HU*, DAILI LAI*, WEI CHEN, SHUMING ZI, JINMING LI, PENG DU, \\ ZHONGCHUAN WANG, SHIYONG HUANG and CUI LONG
}

Department of Colorectal Surgery, Xinhua Hospital Affiliated to Shanghai Jiaotong University School of Medicine, Shanghai 200092, P.R. China

Received March 23, 2011; Accepted June 23, 2011

DOI: $10.3892 / \mathrm{mmr} .2011 .529$

\begin{abstract}
The present study aimed to investigate the expression of the Hedgehog ( $\mathrm{Hh}$ ) signaling pathway between microsatellite-unstable (MSI) and microsatellite-unstable (MSS) colorectal cancers (CRCs). A total of 61 samples of $\mathrm{CRC}$ tissue and corresponding blood samples were obtained from the surgical department of our hospital. The tissue samples were examined by immunohistochemistry using antibodies against Sonic Hh (SHH), Pathed (PTCH) and Gli1, and evaluated independently for protein expression by two pathologists blinded to clinical outcome. Based on the immunohistochemistry results, SHH and PTCH expression varied in terms of histological type. In mucinous adenocarcinoma (MA) Hedgehog signaling was not highly expressed. There were more significant differences in the expression of SHH and PTCH $(\mathrm{P}<0.05)$, compared with Gli1. Moreover, significant differences were found in the expression of $\mathrm{SHH}$, Gli1 and PTCH between the MSI and MSS groups $(\mathrm{P}<0.05)$. Hedgehog signals were more frequently expressed in the MSI group compared with the MSS group. In conclusion, this study indicates that the expression of the Hh signaling pathway may play a significant role in MSI in colorectal cancer.
\end{abstract}

\section{Introduction}

Colorectal cancer (CRC) is one of the most common malignancies worldwide. Despite advances in early detection and improvements in diagnosis and scheduling of therapies in adju-

Correspondence to: Professor Cui Long, Department of Colorectal Surgery, Xinhua Hospital Affiliated to Shanghai Jiaotong University School of Medicine, 1665 Kongjiang Road, Shanghai 200092, P.R. China

E-mail: longcuidr@yahoo.com.cn

*Contributed equally

Key words: Hedgehog signaling pathway, microsatellite instability, colorectal cancer vant and advanced settings, CRC is responsible for substantial mortality and is the leading cause of cancer-related death. CRC exhibits a significant heterogeneity even within the same pathologic stage in both prognosis and response to therapy (1). The clinical heterogeneity may be a sign of underlying molecular heterogeneity in the pathogenesis of CRC. Current knowledge of molecular mechanisms involved in colorectal carcinogenesis indicates that CRC is a multi-pathway disease and two major genomic instability pathways are involved in its pathogenesis: microsatellite instability (MSI) and chromosome instability (CIN). Approximately $15 \%$ of sporadic CRC is characterized by MSI, showing insertions and deletions in DNA microsatellite sequences, whereas the remaining $85 \%$ of CRC develops through the CIN pathway and is characterized by gross chromosomal alterations, either qualitative or quantitative. Although the MSI and CIN phenotypes may be distinguished from one another, evidence indicates some degree of overlap (2).

The Hedgehog (Hh) signaling pathway is known to play a vital role in the proper development of the embryo (3). Thus, the Hh signaling pathway may play a crucial role in tumorigenesis when reactivated in adult tissues (4-8). The involvement of the Hh signaling pathway in CRC has demonstrated that it is essential for human CRC growth, recurrence, metastases and stem cell expansion (9). To gain further insight into the Hh signaling pathway in CRC, we investigated the expression of the Hh signaling pathway between MSI and microsatellitestable (MSS) colorectal cancer.

\section{Materials and methods}

Patients. A total of 61 patients with sporadic CRC were included in this study. Sporadic CRC patients had no known familial history indicative of hereditary non-polyposis colorectal cancer or familial adenoid polyposis. Fresh colorectal carcinomas were obtained from surgical specimens from patients who underwent surgery in the Hospital. Sections from corresponding areas from the specimens submitted for diagnosis were cut into two parts: one part was snap-frozen in liquid nitrogen immediately following resection until processing for DNA isolation, while the other part was formalin-fixed and 
Table I. MSI primer sequence.

\begin{tabular}{llcc}
\hline Locus/Marker & \multicolumn{1}{c}{ Primer Sequence (5'-3') } & Size (bp) & Location \\
\hline BAT-25 & F: TCGCCTCCAAGAATGTAAGT & 124 & 2P16/hMSH2 \\
& R: TCTGCATTTTAATATGGCTC & & $17 \mathrm{q} 11.2-\mathrm{q}$ \\
BAT-26 & F: TGACTACTTTTGACTTCAGCC & 122 & $12 / \mathrm{BRCA}$ \\
& R: AACCATTCAACATTTTAACCC & & $5 \mathrm{q} 21-22 / \mathrm{APC}$ \\
D2S123 & F: AAACAGGATGCCTGCCTTTA & & \\
& R: GGACTTTCCACCTATGGGAC & 210 & $4 \mathrm{q} 12 / \mathrm{C}-\mathrm{kit}$ \\
D5S346 & F: ACTCACTCTAGTGATAAATCGGG & & \\
& R: AGCAGATAAGACAGTATTACTAGT & 115 & \\
D17S250 & F: GGAAGAATCAAATAGACAAT & 152 & \\
& R: GCTGGCCATATATATATTTAAACC & & \\
\hline
\end{tabular}

Table II. The nature, dilution ratio and recommended positive control tissues for each antibody.

\begin{tabular}{lccccc}
\hline Antibody & Nature & Manufacturer & Catalogue no. & Dilution & Positive control tissues \\
\hline SHH & Rabbit monoclonal & Abcam & ab53281 & $1: 100$ & Kidney \\
PTCH & Rabbit polyclonal & Abcam & ab53715 & $1: 50$ & Brain \\
Gli1 & Rabbit polyclonal & Santa Cruz Biotechnology & sc20687 & $1: 50$ & Testis \\
\hline
\end{tabular}

paraffin-embedded for immunohistochemical analysis (IHC). Tumor grading, and assessment of differentiation according to the WHO guidelines, and the immunohistochemical analysis were performed by an experienced pathologist. The experiment acquired a license agreement from the department of hospital ethics.

MSI analysis. Genomic DNA was extracted from each tumor and corresponding blood samples were obtained using a commercial kit (TIANamp Genomic kit, Tiangen Biotech Co., Ltd., Bingjing, China, Catalogue no DP304) according to the manufacturer's instructions. DNA was quantified by nanodrop 2000 to ensure that A260/A280 was between 1.8-1.9. MSI was determined by five standard microsatellite markers, as recommended by the National Cancer Institute Workshop on MSI [the mononucleotide loci (BAT-25 and BAT-26), and the dinucleotide loci (D5S346, D17S250 and D2S123)]. Polymerase chain reaction (PCR) analyses were performed using an ABI PRISM 310 Genetic Analyser (Applied Biosystems, Foster City, CA, USA) and Genescan software (Applied Biosystems). PCR primers are shown in Table I. Laser fluorescence FAM was labeled on each pair's $\mathrm{F}$ primer. A total amount of $2 \mu \mathrm{l}$ genomic DNA from blood samples and tumor tissue in each case was mixed with $2 \mu \mathrm{l}$ primer pairs for the selected microsatellite markers, and $2 \mu \mathrm{l} \mathrm{d}$ NTP Mix, $2 \mu \mathrm{l} 10 \mathrm{X}$ buffer and $0.2 \mu \mathrm{l} \mathrm{Taq}$ water were added to produce a final volume of $20 \mu$ l. The PCR conditions were: after an initial 2-min denaturation step at $95^{\circ} \mathrm{C}, 40$ amplification cycles were performed, each consisting of a 30 -sec step at $95^{\circ} \mathrm{C}$, at $55^{\circ} \mathrm{C}$, and a 30 -sec elongation step at $72^{\circ} \mathrm{C}$. Amplification was completed with a final incubation step at $72^{\circ} \mathrm{C}$ for $7 \mathrm{~min}$. MSI was defined by the presence of novel peaks or loss of bands, and shift of the peak following the PCR amplification of tumor DNA that were not present in normal DNA. A tumor was defined as high-MSI (MSI-H) if more than one of the five examined loci showed unequivocal instabilities. Tumors were classified as MSS if none or only one of the markers exhibited a shift.

Immunohistochemical analysis (IHC). All tissues embedded in paraffin were sliced into $4-\mu \mathrm{m}$ sections. The tissue were deparaffinized with xylene, rehydrated in a serial dilution of alcohol and immersed in $3 \%$ hydrogen peroxide to inhibit endogenous peroxidase activity. The sections were then incubated with various types of primary antibodies overnight at $4^{\circ} \mathrm{C}$. The nature, dilution ratio and recommended positive control tissues of the primary antibodies are shown in Table II. Slides were given three 5-min washes in phosphate-buffered saline (PBS) and incubated with the secondary antibodies and DAB color reagent (Supervision ${ }^{\mathrm{TM}}$ anti-rabbit detection reagent, Shanghai Changdao Biotech, Shanghai, China). The slides were counterstained with Mayer's hematoxylin, then dehydrated, and mounted with Canada balsam for examination. A recommended positive control tissue was used as the positive control for primary antibodies. Staining without the primary antibody was used as a negative control.

Immunohistochemical (IHC) scoring. The slides were evaluated independently for protein expression by two pathologists blinded to clinical outcome. When evaluations differed, a consensus interpretation was achieved using a 2-headed micro- 


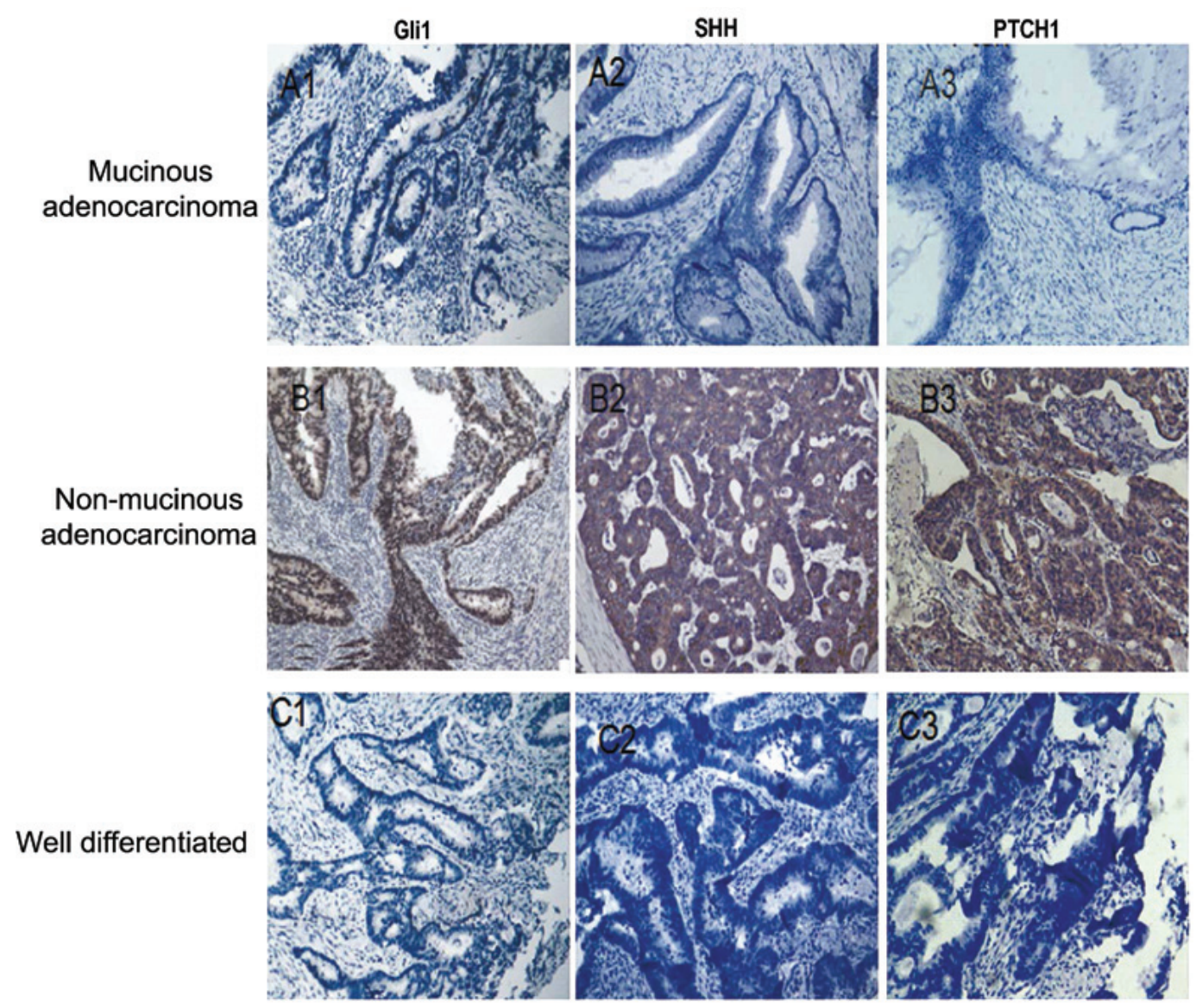

Figure 1. (A1-C1) Immunostaining of Gli1; (A2-C2) immunostaining of SHH; and (A3-C3) immunostaining of PTCH1. Almost no immunoreactivity of HH signal was observed in mucinous adenocarcinomas and well-differentiated carcinomas (A1-C1, A3-C3).

Table III. Patient characteristics.

\begin{tabular}{lccc}
\hline & $\begin{array}{c}\text { MSI } \\
\text { (No. \% })\end{array}$ & $\begin{array}{c}\text { MSS } \\
(\text { No. } \%)\end{array}$ & P-value \\
\cline { 2 - 4 } Gender & & & \\
Female & $7(58.3)$ & $21(42.9)$ & 0.336 \\
Male & $5(41.7)$ & $28(57.1)$ & \\
Age (year) & & & \\
$<50$ & $1(8.30)$ & $5(10.2)$ & 1.000 \\
$>50$ & $11(91.7)$ & $44(89.8)$ & \\
TNM stage & & & \\
I & - & $7(14.3)$ & \\
II & $7(58.3)$ & $21(42.9)$ & \\
III & $5(41.7)$ & $16(32.7)$ & \\
IV & - & $5(10.2)$ & \\
Location & & & \\
Colon & & & \\
Rectum & $7(58.3)$ & $21(42.9)$ & 0.527 \\
Differentiation & $5(41.7)$ & $27(55.1)$ & \\
Poor & & & \\
Moderate & $3(25.0)$ & $6(12.2)$ & \\
Well & $9(75.0)$ & $38(77.6)$ & \\
\hline
\end{tabular}

TNM, tumor, node and metastasis. scope. Only cytoplasmic or nuclear staining was considered for analysis. Evaluation of the staining reaction was performed in accordance with the immunoreactive score (IRS) : IRS = SI (staining intensity) x PP (percentage of positive cells). SI was determined as: 0 , negative; 1 , weak; 2 , moderate; and 3, strong. PP was defined as: 0, negative; 1, 1-10\%; 2, 11-50\%; 3, 51-80\%; and 4, $>80 \%$ positive cells. The combined IRS value was calculated as PP x SI (maximum value was 12). Tumor slices scoring at least 3 points in our study were classified as immunoreactive, indicating a positive expression.

Statistical analysis. SPSS software 15.0 (SPSS, Chicago, USA) was used for statistical analyses. Numeration data were analyzed using the Chi-square or Fisher exact tests as appropriate. A twotailed $\mathrm{P}<0.05$ was considered to be statistically significant.

\section{Results}

MSI status. In total, 12 of $61(19.7 \%)$ tissue samples harbored MSI (Fig. 1). Samples were then divided into MSI and MSS groups in order to analyze the IHC. Comparison of patient characteristics is shown in Table III. No significant differences were noted between MSI and MSS groups (all P>0.05).

Expression of Hh signaling pathway. The expression of $\mathrm{SHH}$ and PTCH was defined as cytoplasmic staining with a stippled or granular pattern. Gli1 was located in cytoplasm and/or 
Table IV. Comparison of the expression of Hedgehog signaling pathway among histological type, differentiation and stage (TNM).

\begin{tabular}{|c|c|c|c|c|c|c|c|c|c|c|}
\hline & \multicolumn{2}{|c|}{ Histological type } & \multirow[t]{2}{*}{ P-value } & \multicolumn{3}{|c|}{ Differentiation } & \multirow[t]{2}{*}{ P-value } & \multicolumn{2}{|c|}{ Stage (TNM) } & \multirow[t]{2}{*}{ P-value } \\
\hline & $\begin{array}{c}\text { NMA } \\
\text { No. }(\%) \\
\end{array}$ & $\begin{array}{c}\text { MA } \\
\text { No. }(\%) \\
\end{array}$ & & $\begin{array}{c}\text { Poor } \\
\text { No. }(\%)\end{array}$ & $\begin{array}{l}\text { Moderate } \\
\text { No. }(\%)\end{array}$ & $\begin{array}{c}\text { Well } \\
\text { No. }(\%)\end{array}$ & & I, II & III, IV & \\
\hline \multicolumn{11}{|l|}{ SHH } \\
\hline Negative & $21(38.9)$ & $7(100)$ & 0.003 & $6(75.0)$ & $18(37.5)$ & $4(100)$ & 0.006 & 20 & 8 & 0.041 \\
\hline Positive & $33(61.1)$ & $0(0)$ & & $2(25.0)$ & $30(62.5)$ & $0(0)$ & & 15 & 18 & \\
\hline \multicolumn{11}{|l|}{ Gli1 } \\
\hline Negative & $24(44.4)$ & $4(57.1)$ & 0.693 & $4(50.0)$ & $22(45.8)$ & $2(50.0)$ & 0.967 & 16 & 12 & 1.000 \\
\hline Positive & $30(55.6)$ & $3(42.9)$ & & $4(50.0)$ & $26(54.2)$ & $2(50.0)$ & & 19 & 14 & \\
\hline \multicolumn{11}{|l|}{ PTCH } \\
\hline Negative & $16(29.6)$ & $7(100)$ & 0.001 & $6(75.0)$ & $13(27.1)$ & $4(100)$ & 0.001 & 16 & 12 & 1.000 \\
\hline Positive & $38(70.4)$ & $0(0)$ & & $3(25.0)$ & $35(72.9)$ & $0(0)$ & & 19 & 14 & \\
\hline
\end{tabular}

NMA, non-mucinous adenocarcinama; MA, mucinous adenocarcinoma; TNM, tumor, node and metastasis.

Table V. Comparison of the expression of Hedgehog signaling pathway between MSI and MSS groups.

\begin{tabular}{lccc}
\hline & MSI & MSS & P-value \\
& No. $(\%)$ & No. $(\%)$ & \\
\hline SHH & & & \\
Positive & $11(91.7)$ & $22(44.9)$ & 0.004 \\
Negative & $1(8.3)$ & $27(55.1)$ & \\
Gli1 & & & \\
Positive & $10(83.3)$ & $23(46.9)$ & 0.028 \\
Negative & $2(16.7)$ & $26(53.1)$ & \\
PTCH & & & \\
Positive & $10(83.3)$ & $27(55.1)$ & 0.022 \\
Negative & $2(16.7)$ & $22(44.9)$ & \\
\hline
\end{tabular}

nucleus but predominatly in cytoplasm (Fig. 1). The positive frequency of SHH and Gli1 in all the cases was $54.1 \%(33 / 61)$, with SHH being higher at $62.3 \%(38 / 61)$. We divided these into positive and negative groups, and undertook an analysis of differentiation, stage, and histological type between the two groups (Table IV). SHH was positively expressed (61.1\%, $33 / 54$ ) in more than half of the non-mucinous adenocarcinoma (NMA) samples, but was not expressed in mucinous adenocarcinoma (MA). This tendency was observed for PTCH, but not Gli1. In MA neither SHH nor PTCH were positive, but Gli1 was partly expressed. With regard to tumor differentiation, $\mathrm{SHH}$ and PTCH were expressed more highly in moderately differentiated tumors than poorly and well-differentiated tumors (SHH $62.5 \%$ vs. $25 \%$ and $0 \%, \mathrm{P}<0.005$; PTCH $72.9 \%$ vs. $25 \%$ and $0 \%, \mathrm{P}<0.005)$, but not Gli1 (54.6\% vs. $50 \%, \mathrm{P}>0.05)$. This differentiation revealed that the expression of SHH and PTCH was significantly correlated to histological type and tumor differentiation, whereas Gli1 expression was not correlated with these clinicopathological variables. Additionally, $\mathrm{SHH}$ expression exhibited significant differences during TNM stages. (Table IV).

Significant differences were noted in the expression of the three antibodies between the MSI and MSS groups. In general, SHH, Gli1 and PTCH were more frequently expressed in the MSI group compared with the MSS group. Comparison of the expression of Hh signaling pathway is shown in Table V.

\section{Discussion}

MSI in CRC is mainly caused by mutations in the DNA mismatch repair genes hMLH1 and hMSH2, whereas the expression of hMSH6, hPMS1 and hPMS2. The mechanism of tumorigenesis in MSI-H tumors is thought to involve frameshift mutations of microsatellite repeats within coding regions of the affected target genes, and the inactivation of these target genes is believed to directly contribute to tumor development and progression (10).

A common concept, MSI-H identifies a well-defined subset of CRC that tends to exhibit a diploid state, to be more proximal, poorly differentiated and mucinous, and to have marked lymphocyte infiltration (11). Thus, MSI genotyping may allow for the identification of discrete molecular CRC subtypes, and MSI appears to be one of the most promising molecular markers with both prognostic and predictive value for chemosensitivity (12). MSI testing may also result in larger numbers of patients being assigned the appropriate treatment based on their disease profile.

$\mathrm{Hh}$ signaling is required for the normal growth and regeneration of organs, such as lung, gastrointestinal tract and prostate, from stem cells. Inappropriate and constitutive activation of the Hedgehog pathway during tissue repair and regeneration may promote tumorigenesis (13). The $\mathrm{SHH}$ signaling pathway may play a central role in the pathogenesis of CRC and the maintenance of Hh signaling pathways for CRC tumorigenesis $(14,15)$. In certain tumors, such as basal cell carcinoma and central nervous system tumors, active muta- 
tions of SHH, Smo and loss of function mutations of PTCH may activate the Hh pathway $(13,16,17)$. The Hh pathway plays a significant role in hepatocellular carcinoma development and invasion (18). We observed that Hedgehog was much less frequently expressed in MA than in NMA. Previous studies indicated that MSI-H gene alterations were more common in MA than in NMA (19). Further studies are required to analyze this phenomenon. In our study, $\mathrm{SHH}$ and $\mathrm{PTCH}$ were more frequently expressed in the MSI group compared with the MSS group. SHH activation was associated with downstream activation of Gli1 and FOXM1 transcription factors known to promote cell proliferation through the induction of the cyclin genes $(20,21)$. Gli1 level and activity may thus be modulated by multiple oncogenes and the environment of the cancer cells (22). We may hypothesize that some of these genes are involved in the Hh signal pathway. However, further analysis of this hypothesis is required. However, whether or not other signaling pathways were involved in impacting the Hh components or mediating the genes in the network of tumorigenesis remains to be determined.

The molecular dissection of genes differently expressed in colorectal cancer was provoked by the knowledge that MSI-H colorectal carcinomas are distinguished from MSS carcinomas. Previous studies using oligonucleotide microarray analysis have revealed that there are distinct gene expression profiles in MSI-H and MSS colorectal carcinomas (23). Although the involvement of the Hh signaling pathway in CRC remains controversial, this is the first time that expression of the Hh signaling pathway has been analyzed in MSS and MSI, demonstrating a molecular signature responsible for CRC. Our study indicates that the expression of the Hh signaling pathway may play a crucial role in MSI for colorectal cancer. However, this mechanism requires further investigation.

\section{Acknowledgements}

This study was supported by the Science and Technology Commission of Shanghai Municipality through a grant-in-aid for key projects of basic research (09JC1410900) and major projects of basic research (10DJ1400504).

\section{References}

1. Jass JR: Molecular heterogeneity of colorectal cancer: implications for cancer control. Surg Oncol 16 (Suppl 1): 7-9, 2007.

2. Grady WM and Markowitz S: Genomic instability and colorectal cancer. Curr Opin Gastroenterol 16: 62-67, 2000.

3. Ingham PW and McMahon AP: Hedgehog signaling in animal development: paradigms and principles. Genes Dev 15:3059-3087, 2001.

4. Kim Y, Yoon JW, Xiao XK, Dean NM, Monia BP and Marcusson EG: Selective down-regulation of glioma-associated oncogene 2 inhibits the proliferation of hepatocellular carcinoma cells. Cancer Res 67: 3583-3593, 2007.

5. Cutcliffe C, Kersey D, Huang CC, Zeng Y, Walterhouse D and Perlman EJ: Clear cell sarcoma of the kidney: up-regulation of neural markers with activation of the sonic hedgehog and Akt pathways. Clin Cancer Res 11: 7986-7994, 2005.
6. Liao X, Siu MK, Au CW, et al: Aberrant activation of hedgehog signaling pathway in ovarian cancers: effect on prognosis, cell invasion and differentiation. Carcinogenesis 30: 131-140, 2009.

7. Bailey JM, Swanson BJ, Hamada T, et al: Sonic hedgehog promotes desmoplasia in pancreatic cancer. Clin Cancer Res 14: 5995-6004, 2008.

8. Watkins DN, Berman DM, Burkholder SG, Wang BL, Beachy PA and Baylin SB: Hedgehog signalling within airway epithelial progenitors and in small-cell lung cancer. Nature 422: 313-317, 2003.

9. Varnat F, Duquet A, Malerba M, Zbinden M, Mas C, Gervaz P and Ruiz i Altaba A: Human colon cancer epithelial cells harbour active HEDGEHOG-GLI signalling that is essential for tumour growth, recurrence, metastasis and stem cell survival and expansion. Embo Mol Med 1: 338-351, 2009.

10. Abdel-Rahman WM, Mecklin JP and Peltomaki P: The genetics of HNPCC: application to diagnosis and screening. Crit Rev Oncol Hematol 58: 208-220, 2006.

11. Soreide K, Janssen EA, Soiland H, Korner H and Baak JP: Microsatellite instability in colorectal cancer. Br J Surg 93: 395-406, 2006.

12. Muller CI, Schulmann K, Reinacher-Schick A, et al: Predictive and prognostic value of microsatellite instability in patients with advanced colorectal cancer treated with a fluoropyrimidine and oxaliplatin containing first-line chemotherapy. A report of the AIO Colorectal Study Group. Int J Colorectal Dis 23: 1033-1039, 2008.

13. Evangelista M, Tian $\mathrm{H}$ and de Sauvage FJ: The hedgehog signaling pathway in cancer. Clin Cancer Res 12: 5924-5928, 2006.

14. Luebeck EG and Moolgavkar SH: Multistage carcinogenesis and the incidence of colorectal cancer. Proc Natl Acad Sci USA 12: 15095-15100, 2002.

15. Ohta M, Tateishi K, Kanai F, et al: p53-Independent negative regulation of $\mathrm{p} 21 / \mathrm{cyclin}$ dependent kinase interacting protein 1 by the sonic hedgehog glioma associated oncogene 1 pathway in gastric carcinoma cells. Cancer Res 65: 10822-10829, 2005.

16. Nakashima $H$, Nakamura $M$ and Yamaguchi $H$, et al: Nuclear Factor-kappa B contributes to hedgehog signaling pathway activation through sonic hedgehog induction in pancreatic cancer. Cancer Res 66: 7041-7049, 2006.

17. Cutclife C, Kersey D, Huang CC, Zeng Y, Walterhouse D and Perlman EJ: Clear cell sarcoma of the kidney: up-regulation of neural markers with activation of the sonic hedgehog and Akt pathways. Clin Cancer Res 11: 7986-7993, 2005.

18. Cheng WT, Xu K, Tian DY, Zhang ZG, Liu LJ and Chen Y: Role of Hedgehog signaling pathway in proliferation and invasiveness of hepatocellular carcinoma cells. Int J Oncol 34: 829-836, 2009.

19. Song GA, Deng GR, Bell I, Kakar S, Sleisenger MH and Kim YS: Mucinous carcinomas of the colorectum have distinct molecular genetic characteristics. Int J Oncol 26: 745-750, 2005.

20. Duman-Scheel M, Weng L, Xin S and Du W: Hedgehog regulates cell growth and proliferation by inducing Cyclin D and Cyclin E. Nature 417: 299-304, 2002.

21. Wang X, Kiyokawa H, Dennewitz MB and Costa RH: The Forkhead Box $\mathrm{m} 1 \mathrm{~b}$ transcription factor is essential for hepatocyte DNA replication and mitosis during mouse liver regeneration. Proc Natl Acad Sci USA 99: 16881-16886, 2002.

22. Varnat F, Siegl-Cachedenier I, Malerba M, Geruaz P and Ruiz i Altaba A: Loss of WNT-TCF addiction and enhancement of HH-GLI1 signaling define the metastatic transition of human colon carcinomas. EMBO Mol Med: 2440-2457, 2010.

23. Kim H, Nam SW, Rhee H, et al: Different gene expression profiles between microsatellite instability-high and microsatellite stable colorectal carcinomas. Oncogene 23: 6218-6225, 2004. 